\title{
Genomics of maize resistance to kernel contamination with fumonisins using a multiparental advanced generation InterCross maize population (MAGIC)
}

\author{
Noemi Gesteiro ${ }^{1 *}$, Ana Cao ${ }^{1}$, Rogelio Santiago ${ }^{2}$, Rosa Ana Malvar ${ }^{1}$ and Ana Butrón ${ }^{1}$
}

\begin{abstract}
Maize kernel is exposed to several fungal species, most notably Fusarium verticillioides, which can contaminate maize kernels with fumonisins. In an effort to increase genetic gains and avoid the laborious tasks of conventional breeding, the use of marker-assisted selection or genomic selection programs was proposed. To this end, in the present study a Genome Wide Association Study (GWAS) was performed on 339 RILs of a Multiparental Advanced Generation InterCross (MAGIC) population that had previously been used to locate Quantitative Trait Locus (QTL) for resistance to Fusarium Ear Rot (FER). Six QTLs for fumonisin content were detected in the bins 3.08, 4.07, 4.10, 7.03-7.04, 9.04-9.05 and 10.04-10.5. Five of the six QTLs collocate in regions where QTLs for FER were also found. However, the genetic variation for fumonisin content in kernel is conditioned by many other QTLs of small effect that could show QTLx environment interaction effects. Although a genomic selection approach to directly reduce fumonisin content in the kernel could be suitable, improving resistance to fumonisin content by genomic selection for FER would be more advisable.
\end{abstract}

Keywords: Maize kernel, Fumonisin, Resistance, MAGIC, GWAS

\section{Background}

Maize kernel is exposed to several fungal genera, including Fusarium, Aspergillus or Penicillium, which can contaminate maize kernels with mycotoxins. In Spain, Fusarium verticillioides predominates over other species. This species produces fumonisins that, in addition to causing multiple disorders in animals [1], have been classified as possibly carcinogenic by the International Agency for Research on Cancer [2].

In order to increase genetic gains and eliminate expensive, labor and time-consuming tasks associated to conventional breeding, such as artificial inoculations and

\footnotetext{
${ }^{*}$ Correspondence: ngesteiro@mbg.csic.es

${ }^{1}$ Misión Biológica de Galicia (CSIC), Box 28, 36080, Pontevedra, Spain

Full list of author information is available at the end of the article
}

fumonisin quantifications, markers linked to the genes involved in resistance to fumonisin contamination can be used in marker-assisted selection programs. Therefore, many studies have been focused on the detection of Quantitative Trait Locus (QTL) for resistance to Fusarium Ear Rot (FER) [3-8], but fewer locate QTL for resistance to fumonisin contamination [3, 7-9]. FER can be scored on a visual scale, whereas kernel fumonisin quantification is more laborious and expensive, requiring high-performance chromatography or enzyme-linked immunosorbent assays (ELISA). Although screening for FER would be an effective way to improve resistance to fumonisin contamination due to moderate to high correlation coefficients between both traits [10-14], QTL studies for fumonisin content are still necessary because 
that association has been noted to be weaker in particular genetic backgrounds [13].

Until now, most QTL studies for fumonisin content were done using biparental mapping populations $[3,7$, 8]. In recent years, however, mapping approaches involving multiparental designs such as the Nested Association Mapping (NAM) proposed by Yu et al. [15] and Multiparental Advanced Generation InterCross (MAGIC) proposed for crop mapping by Mackay et al. [16] have gained attention. In contrast to inbred panels, multiparental populations largely avoid the potentially confounding influence of population structure and increase the frequency of panel rare alleles which could be of particular interest for breeding [17]. An efficient MAGIC population has clear advantages over other approaches: it has greater genetic variation than biparental populations; it has a balanced allelic frequency since all founders contribute equally; and a uniform and high recombination rate that increases the resolution of genetic analysis, mapping and gene isolation [18].

In the current study, a genome-wide association study (GWAS) approach has been used to find QTL for resistance to fumonisin contamination in an eight-way MAGIC population previously used for locating QTL for FER [10]. In this MAGIC population founders have shown high diversity for specific traits, FER and fumonisin content among those traits [10, 19-25]. In addition, MAGIC populations could be used to obtain superior breeding lines for resistance to FER and to fumonisin contamination [26], or as base materials to perform genomic selection [27]. Thus, the main objectives of this research were: (i) to carry out a GWAS analysis in a MAGIC population in order to identify novel QTLs involved in reduced fumonisin contamination in the maize kernel, and (ii) to propose the most suitable breeding program to reduce kernel contamination with fumonisins.

\section{Results}

Founders were profusely replicated in 2014 trial (16 replicates) meanwhile, in 2016, they were just replicated twice in a trial with 800 plots (rows). Therefore, the estimation of founder mean was considerably less precise in 2016 and no significant differences were found among them in that year. However, significant differences were found among founders for fumonisin content in kernels in $2014(\mathrm{~F}=5.6, P>0.0001)$ (Fig. 1). Data on fumonisin content of each RIL in 2014 and 2016 are shown in Additional file 1 . The genetic heritability for kernel fumonisin content estimated on a mean basis, although low due to significant genotype $\mathrm{x}$ environment interaction $\mathrm{Z}=7.28$, $p<0.0001)$, was significantly different from zero $(0.37 \pm 0.067)$. The phenotypic correlation coefficient between fumonisin content and FER $(0.57 \pm 0.03)$ was moderate and significant, while the genotypic correlation coefficient was high $(0.92 \pm 0.09)$. Neither significant genotypic $(-0.07 \pm 0.11)$ and phenotypic $(-0.06 \pm 0.04)$ correlation coefficients were found between fumonisin content and days to silking.

Twenty-four SNPs could be considered as putatively linked to QTLs because they were located at the top of the Q-Q plot and presented $p$ values $<0.0001$ (Fig. 2); those observed $p$ values being significantly lower than

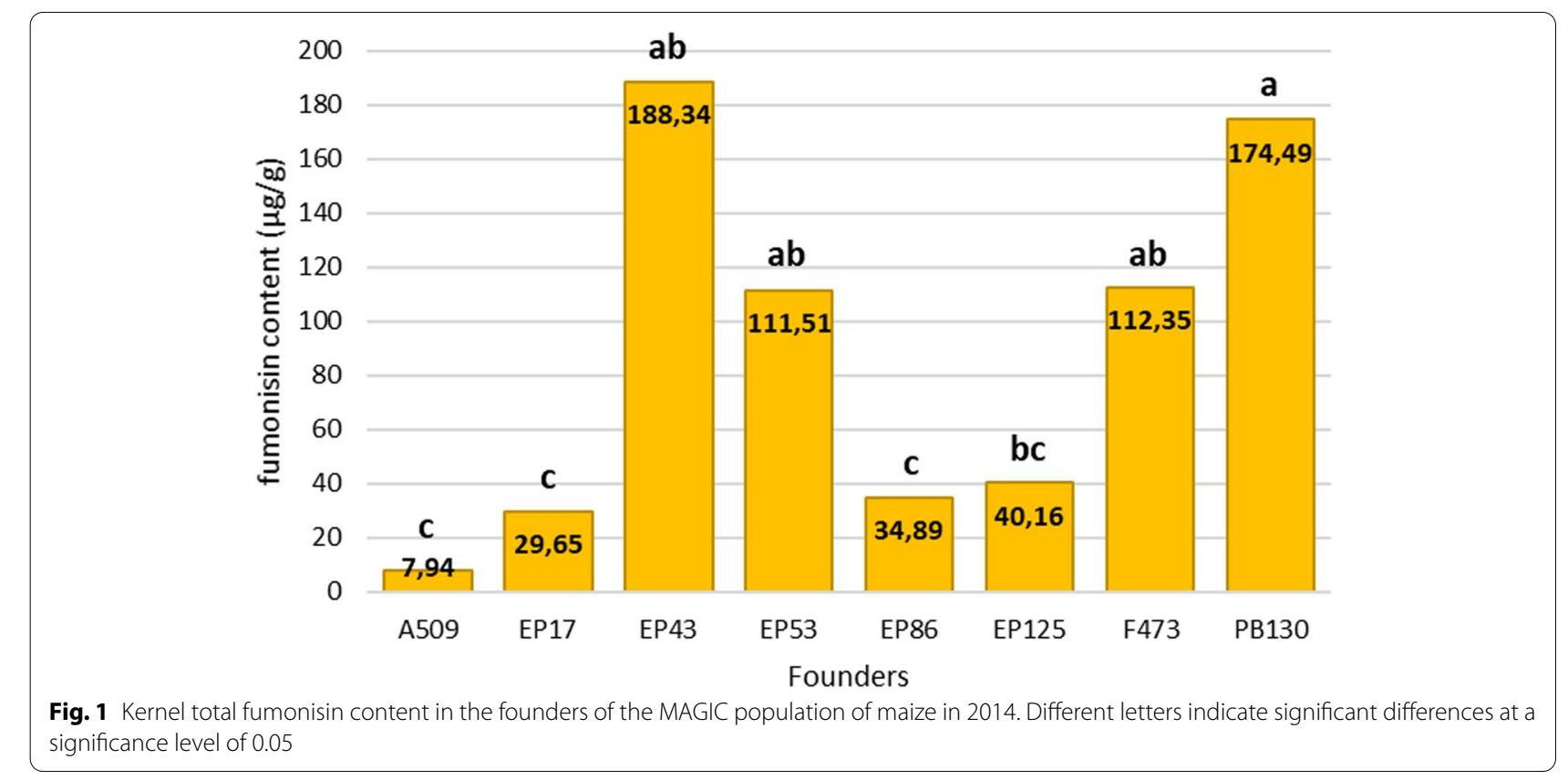




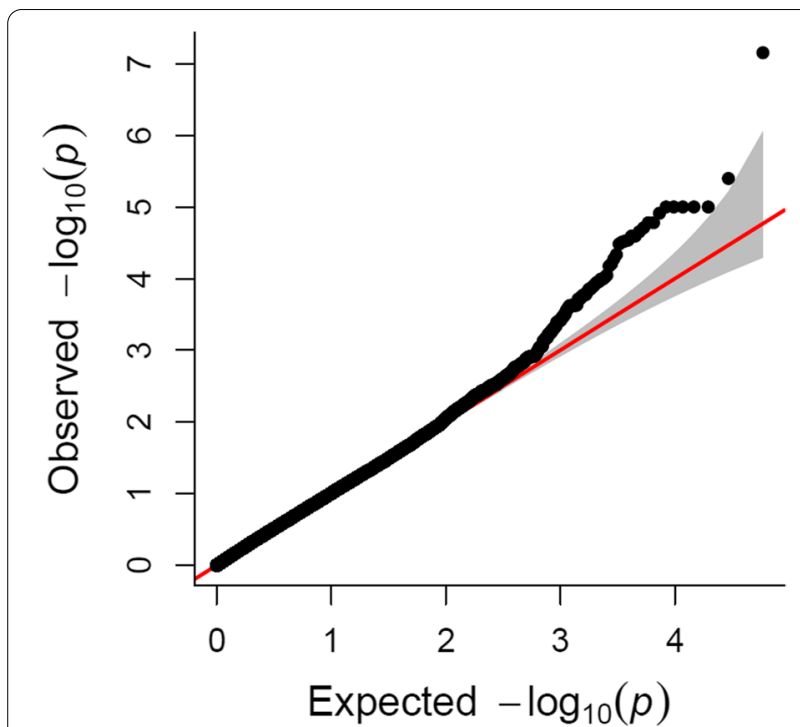

Fig. 2 Quantile-quantile plots of a mixed linear model for kernel fumonisin content in the MAGIC population. The dotted lines show the $95 \%$ confidence interval for the QQ-plot under the null hypothesis of no association between the SNP and kernel fumonisin content

those expected under no significant association between SNPs and fumonisin content. However, only one of these outliers exceeded the alpha $=0.10$ genome-wide $p$-value threshold (empirically estimated as a comparison-wide $p$-value of 7.02E-08; Fig. 3). The approximate support interval for each significant SNP was established by visually checking the linkage disequilibrium (LD) heatmap plot of the genomic region containing the SNP. The support interval was considered to be the region where appreciable LD was observed between SNPs $\left(r^{2}>0.3\right)$. Supporting intervals for QTLs were generally less than $20 \mathrm{Mbp}$, except for QTLs located at or near centromeres, where LD is exceptionally high.

Six putative QTLs were found for fumonisin content at bins 3.08, 4.07, 4.10, 7.03-7.04, 9.04-9.05 and 10.0410.5 because SNPs with overlapping confidence intervals were clustered in the same QTL. No appreciable LD $\left(\mathrm{r}^{2}>0.2\right)$ was found between SNPs located in different QTLs (Fig. 4). The most reliable QTL would be at 235$237 \mathrm{Mb}$ on chromosome $4\left(p\right.$ value $\left.=7.0^{-08}\right)$ (Table 1$)$. As this region comprises only $2 \mathrm{Mb}$ all genes contained in it were considered as candidate genes but the discussion will focus only in Zm00001d053751 with an annotated function possibly involved in resistance. In addition, it should be noted that, at significant SNPs, the frequencies of unfavorable alleles for kernel fumonisin accumulation were lower than those of favorable alleles.

\section{Discussion}

Large and significant differences among inbred founders were detected for fumonisin content, but the estimated heritability for fumonisin content in the MAGIC population derived from those founders was low highlighting the importance of moving to breeding methodologies assisted by molecular markers with stable effects across environments. Appreciable additive genetic variability among the RILs of the MAGIC population was found because the heritability estimate, although low, was significantly different from zero and similar to those obtained for other genetically diverse populations $[7,9$,

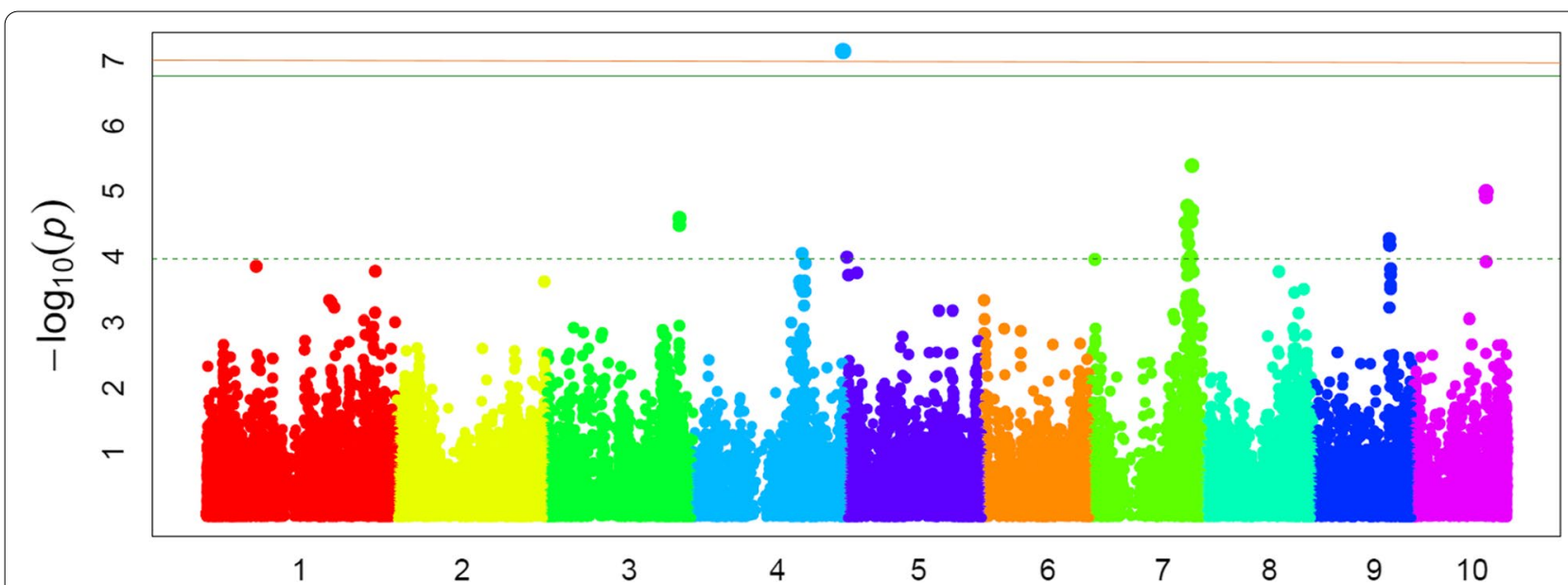

Fig. 3 Manhattan plot of a mixed linear model for kernel fumonisin content in the MAGIC population. Single nucleotide polymorphisms (SNPS) above the green horizontal line exceeded the Bonferroni limit established for an experiment-wide error of 0.01 (default threshold supplied by Farm(PU), above the red horizontal line exceeded the alpha $=0.10$ genome-wide $p$-value threshold and above the dotted horizontal line the $(p$ value $=0.0001$ ) samples fell outside the $95 \%$ confidence interval for no significant association between SNP and fumonisin content 


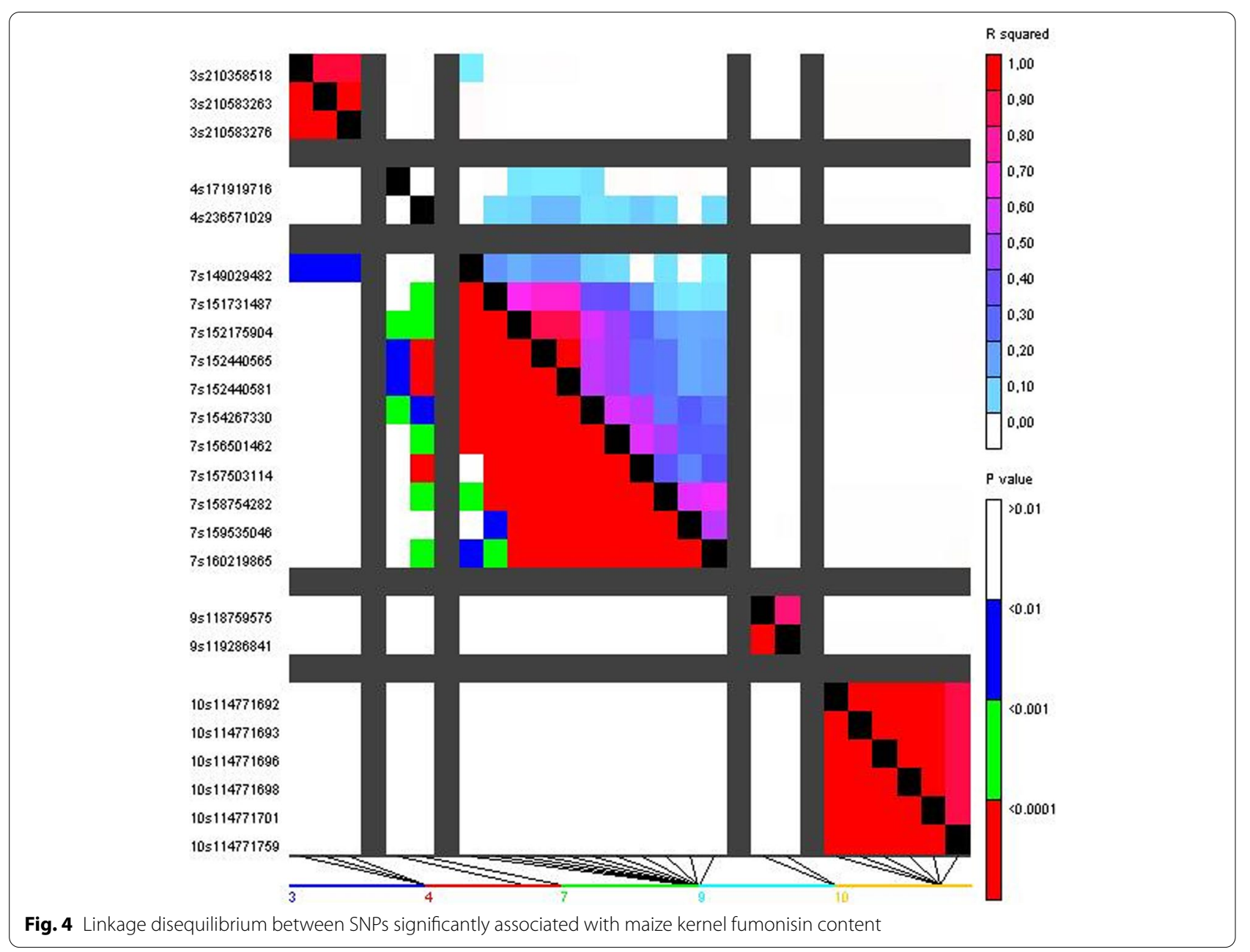

12, 14, 28]. These results also confirmed that the kernel inoculation technique would be suitable to detect differences for fumonisin content among genotypes, probably because would guarantee a more homogeneous dispersal of the inoculum across genotypes than other inoculation techniques $[8,29,30]$.

In the current MAGIC population, genetic variation for kernel fumonisin content was not associated to variability for days to silking as it often occurred among other materials $[3,7,9]$ Therefore, breeding for resistance to fumonisin content in this MAGIC population would not result in unintended maturity changes that would compromise adaptation to specific environments, an important advantage over other mapping populations. The smaller linkage blocks and reduced population structure of this MAGIC population compared to bi-parental populations and association panels, respectively, could allow independent segregation of both traits [10].

According to other studies, kernel fumonisin content was highly correlated with FER and, consequently, colocalizations between QTLs for FER and fumonisin content were expected [9-12, 14]. Five out of the six QTLs found for fumonisin content were located in genomic regions where QTLs for FER were found in a previous study using the same population [10]. However, there are studies in which high concentrations of fumonisins were found in visually FER resistant genotypes and, in those cases, the QTL conferring resistance/susceptibility to fumonisin contamination would not show any effect on FER [12, 13, 31]. According to these observations, the most reliable QTL for fumonisin content was found in a region where no QTL for FER were previously detected [10].

Among genes contained in the supporting interval of the QTL at bin 4.10, a gene annotated as negative regulator of systemic acquired resistance (SNI1),

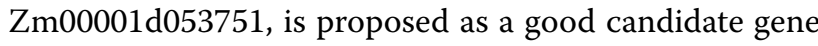
for that QTL. In Arabidopsis, disruption of SNI1 function resulted in enhanced sensitivity to salicylic acid and increased resistance response to pathogens; SNI1 being 


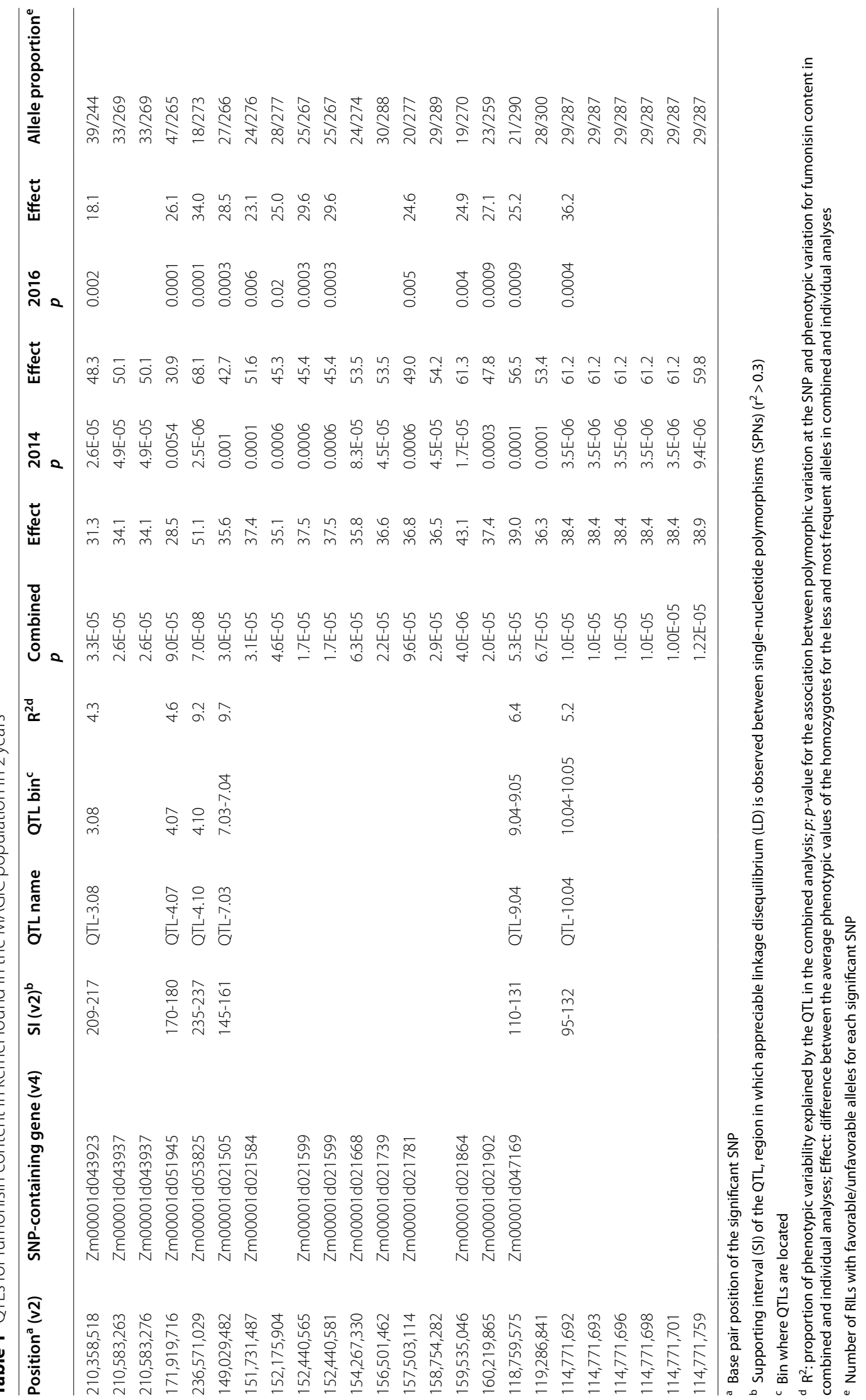


considered as a subunit of the SMC5/6 complex that plays critical roles in DNA damage responses including DNA damage repair [32, 33]. In addition, Wang et al. [34] showed that SNI1 is a negative regulator of E2F transcriptor factors and could play dual roles in DNA damage responses by linking cell cycle checkpoint and DNA repair. However, recent investigations have showed that SNI1 is not directly involved in systemic acquired resistance or DNA damage accumulation, as it was previously proposed, but have suggested that the gene sni1 could be involved in regulating or signaling immunity $[35,36]$. Sanchez-Rangel et al. [37] suggested that activation of the salicylic acid pathway will induce cell death and could facilitate necrotroph proliferation of Fusarium verticillioides. Therefore, as SNI1 is involved in salicylic acidmediated response, we hypothesize that the snil gene could have effect on fumonisin content without affecting FER because it could be involved in conditioning the change from biotrophic to necrotrophic behavior of the fungus.

Many small-effect QTLs appear to be involved in maize resistance to fumonisin contamination, so the value of targeting the significant SNPs to improve resistance to fumonisin contamination of grain is limited because each SNP explains a small percentage of variation [7, 38, 39]. In accordance with that, marker assisted selection based on the QTLs found in the current study, although can serve to discard genotypes extremely contaminated, could not support the pyramiding of minor favorable alleles whose effects were probably hidden by residual and QTL $x$ environment variation. However, genes behind the QTL detected could deserve to be positionally cloned as they seemed to have important effects on fumonisin content across populations $[3,7,9]$. Although the search of QTLs directly involved in resistance to fumonisin contamination uncovered a QTL which was not found when looking for QTLs for FER, lowest stability of genotypes across environments for fumonisin content was observed compared to FER (family- based heritabilities for FER and fumonisin content were 0.56 and 0.37 , respectively) [10]. The plot estimate for FER was the average of five individual (ears) values [10], whereas fumonisin content was determined in a sample of the flour obtained from those ears and distribution of mycotoxins in maize flour is not totally homogeneous $[40,41]$. Therefore, the data collection itself may contribute to those differences between heritability estimates because lower accuracy for fumonisin content than for FER is expected.

Marker assisted selection based on the QTLs found in the current study could not support the pyramiding of minor favorable alleles whose effects were probably hidden by residual and QTL $x$ environment variation. Considering that the heritability of fumonisin content is low and that each significant SNP explains a small proportion of the phenotypic variability, a genomic selection program to directly reduce fumonisin content would be appropriate, but indirect selection through genomic selection for FER would be more advisable because genetic correlation between both traits is very high and FER evaluation is more cost efficient.

\section{Conclusions}

In conclusion, the current MAGIC population allows finding QTLs involved in resistance to fumonisin accumulation in maize kernel and, its population structure allows independent segregation of genes favouring resistance enhancement without compromising other aspects. However, genetic variation for fumonisin content in maize kernel is conditioned by genotype $\mathrm{x}$ environment interaction and many small effect QTLs. Although a direct genomic selection approach to reduce fumonisin content could be suitable, improving resistance to fumonisin accumulation by genomic selection for FER could be easier and more cost efficient.

\section{Methods}

A MAGIC population of recombinant inbred lines (RIL) was developed from the synthetic variety EPS21 as described previously $([10,20]$. EPS21 synthetic was composed of eight unrelated inbred lines, which had in common the lack of "Reid" materials in their pedigrees. The inbred lines used to construct the EPS21 synthetic were A509, EP17, EP43, EP53, EP86, EP125, F473 and PB130 that showed contrasting levels of resistance to FER and fumonisin accumulation in kernel in preliminary evaluations [21]. Inbreds EP17, EP86, EP125 and A509 were partially resistant to fumonisin accumulation, while EP43, EP53, F473 and PB130 were susceptible. The set of RILs from the MAGIC population together with the eight parental founders included as testers were evaluated for resistance to kernel fumonisin accumulation in Pontevedra, Spain $\left(42^{\circ} 24^{\prime} \mathrm{N}, 8^{\circ} 38^{\prime} \mathrm{W}, 20 \mathrm{~m}\right.$ above sea level) in 2014 and 2016. In 2014, shortage of seed amount for the MAGIC RILs made us to opt for an experimental design in which those materials were unreplicated, but checks (parental founders of the MAGIC) were profusely replicated to account for block effects. As seed amount for each RIL was increased in 2015, all entries could be replicated in the experimental design used in 2016. In 2014, 672 RILs and the eight founders were evaluated using an augmented design with 16 blocks. The 672 RILs were un-replicated and randomly assigned to 16 blocks. The eight inbred founders were replicated and randomly assigned to plots within each of the 16 blocks, i.e. each block consisted of 42 non-replicated RILs plus the eight founders. The experimental plot consisted of a 13-plant 
row with a spacing of $0.18 \mathrm{~m}$ between plants and $0.8 \mathrm{~m}$ between rows. In 2016, 695 RILs and seven parental founders (no seed from PB130 was available) were evaluated using a $26 \times 27 \alpha$-lattice design with two replications. Each experimental plot consisted of one 19-plant row with a spacing of $0.18 \mathrm{~m}$ between plants and $0.8 \mathrm{~m}$ between rows.

In both years, five primary ears were inoculated in each plot using a kernel inoculation technique with a four-needle vaccinator: approximately 7-14days after silking (when half of the plot plants showed visible pistils or silks), the main ears of five plants were inoculated with $2 \mathrm{ml}$ of a spore suspension $\left(10^{6}\right.$ spores $\left./ \mathrm{ml}\right)$ of an isolate of $F$. verticillioides [42]. Harvesting of each plot was made two months after inoculation. Collected ears were dried at $35^{\circ} \mathrm{C}$ for one week, shelled and a representative kernel sample of approximately $60 \mathrm{~g}$ was ground using a $0.75 \mathrm{~mm}$ sieve in a Pulverisette 14 rotor mill (Fritsch $\mathrm{GmbH}$, Oberstein, Germany). The ground samples were sent to the service "Microbiological Quality in the AgroFood Sector" of the University of Lleida, Spain, for the determination of total fumonisins (fumoninins $B_{1}, B_{2}$ and $\mathrm{B}_{3}$ ) using a commercial ELISA kit (R-Biopharm Rhône Ltd., Glasgow, Scotland, United Kingdom). The recovery rate of the test was approximately $60 \%$ with an average coefficient of variation of approximately $8 \%$; specificities for $B_{1}$ were $100 \%$, for $B_{2}$ approximately $40 \%$ and for $B_{3}$ close to $100 \%$. The detection limit was $0.025 \mathrm{ppm}\left(\mathrm{mg} \mathrm{kg}^{-}\right.$

${ }^{1}$ ). Both sample extraction and preparation, as well as test performance, were carried out as described in the commercial kits.

The GWAS was performed with 339 RILs that presented complete phenotypic data in both years and heterozygosity levels below the $15 \%$. The RILs were previously genotyped for approximately 1000,000 SNPs using a genotyping-by-sequencing (GBS) strategy at the Cornell University Biotechnology Institute. Monomeric and multiallelic SNPs and insertion/deletion polymorphisms were excluded as well as SNPs with more than $20 \%$ of missing data or with allele frequency (MAF) less than $5 \%$. A total of 58,556 filtered SNPs distributed across the maize genome were used for GWAS.

First, a combined analysis of variance for kernel fumonisin content was performed using the PROC MIXED procedure of SAS software with RILs as fixed effects and years, replications, years $\mathrm{x}$ RILs and blocks as random effects. The best linear unbiased estimator (BLUE) was calculated for each RIL in each year and average RIL data across years were used to perform GWAS analysis.

Means for fumonisin content of inbred founders, calculated by the least square procedure, were compared by Fisher's protected least significant difference (LSD) and only data recorded in 2014 were analyzed because inbred founders were feebly replicated in the trial of 2016. The heritability $\left(\mathrm{h}^{2}\right)$ for kernel fumonisin content in the MAGIC population was estimated on a RIL-mean basis [43]. Genotypic $\left(r_{g}\right)$ and phenotypic $\left(r_{f}\right)$ correlations between kernel fumonisin content and previously published data [10] such as FER and days to silking (these data come from the same trials) were calculated following Holland [44].

GWAS analysis based on a linear mixed model was performed using FarmCPU [45]. We calculated an empirical comparison $p$-value threshold using 1000 data permutation runs and assuming a wide-experiment error of 0.10 (7.02E-08). As, in the Q-Q plot (Fig. 2), F test statistics significantly deviated from the expected F test statistics at $p<0.0001$, SNPs with $p$ values lower than 0.0001, although less reliable than those below the empirical threshold, deserve some consideration. The GWAS analysis was completed with an analysis of linkage disequilibrium (LD) between SNPs significantly associated with kernel fumonisin content using the version 5 of TASSEL [46]. A support interval for significant association was established, and all genes contained in support intervals smaller than $2 \mathrm{Mb}$ were considered as candidate genes and identified and characterized using MaizeGBD genome browser [47]. The search for candidate genes was performed on version 4 of the B73 sequence (Zm-B73-REFERENCE-GRAMENE-4.0).

\section{Supplementary Information}

The online version contains supplementary material available at https://doi. org/10.1186/s12870-021-03380-0.

Additional file 1. Average fumonisin content $(\mu \mathrm{g} / \mathrm{g})$ of each MAGIC RIL in trials performed in 2014 and 2016.

\section{Acknowledgments}

NG acknowledges an FPI predoctoral fellowship. AC acknowledges a contract as research-assistant technician (PTA2018-015873-I funded by MCIU/AEI/ FEDER, UE). RS acknowledges postdoctoral contract "Ramón y Cajal" financed by the Ministry of Economy and Competiveness (Spain), Vigo University, and the European Social Fund. Paper publication expenses were covered by the CSIC and the Programa Contrato of the Xunta of Galicia.

\section{Authors' contributions}

NG: statistical analysis, writing - original draft, writing - review \& editing, visualization. AC: coordinated field inoculations, collaborated in data collection and sample processing and read and approved the manuscript. RS: collaborated in data collection and sample processing and read and approved the manuscript. RAM: collaborated in the design of the research and read and approved the manuscript. AB: conceived and designed the experiments, supervised field experiments and statistical analyses and read and approved the manuscript. All authors read and approved the final manuscript

\section{Funding}

This research was funded by a coordinated project [subprojects RTI2018096776-B-C21 and RTI2018-096776-B-C22] financed by MCIU/AEI/FEDER, UE. The sponsor(s) had not involvement in study design; in the collection, analysis and interpretation of data; in the writing of the report; and in the decision to submit the article for publication. 


\section{Availability of data and materials}

The datasets used and/or analysed during the current study are available from the corresponding author on reasonable request.

\section{Declarations}

\section{Ethics approval and consent to participate}

\section{Not applicable.}

\section{Competing interests}

The authors declare that they have no competing interests.

\section{Author details}

${ }^{1}$ Misión Biológica de Galicia (CSIC), Box 28, 36080, Pontevedra, Spain. ${ }^{2}$ Departamento Biología Vegetal y Ciencias del Suelo, Facultad de Biología, Universidad de Vigo, Unidad Asociada Agrobiología Ambiental, Calidad de Suelos y Plantas, As Lagoas Marcosende, 36310 Vigo, Spain.

Received: 22 July 2021 Accepted: 17 November 2021

Published online: 16 December 2021

\section{References}

1. Butrón A, Reid LM, Santiago R, Cao A, Malvar RA. Inheritance of maize resistance to gibberella and fusarium ear rots and kernel contamination with deoxynivalenol and fumonisins. Plant Pathol. 2015;64:1053-60.

2. IARC. Fumonisin B1. Some traditional herbal medicines, some mycotoxins, naphthalene and styrene. In: 82 Monograph of the International Agency for Research of Cancer on the evaluation of carcinogenic risks to humans. Lyon; 2002. p. 301-66.

3. Maschietto V, Colombi C, Pirona R, Pea G, Strozzi F, Marocco A, et al. QTL mapping and candidate genes for resistance to fusarium ear rot and fumonisin contamination in maize. BMC Plant Biol. 2017;17:20.

4. Giomi GM, Kreff ED, Iglesias J, Fauguel CM, Fernandez M, Oviedo MS, et al. Quantitative trait loci for fusarium and Gibberella ear rot resistance in Argentinian maize germplasm. Euphytica. 2016;211:287-94.

5. Chen J, Ding J, Li H, Li Z, Sun X, Li J, et al. Detection and verification of quantitative trait loci for resistance to fusarium ear rot in maize. Mol Breed. 2012;30:1649-56. https://doi.org/10.1007/s11032-012-9748-1.

6. Li ZM, Ding JQ, Wang RX, Chen JF, Sun XD, Chen W, et al. A new QTL for resistance to fusarium ear rot in maize. J Appl Genet. 2011:52:403-6. https://doi.org/10.1007/s13353-011-0054-0.

7. Robertson-Hoyt LA, Jines MP, Balint-Kurti PJ, Kleinschmidt CE, White DG, Payne GA, et al. QTL mapping for fusarium ear rot and fumonisin contamination resistance in two maize populations. Crop Sci. 2006:46:1734-43.

8. Morales L, Zila CT, Moreta Mejía DE, Montoya Arbelaez M, Balint-Kurti PJ, Holland JB, et al. Diverse components of resistance to fusarium verticillioides infection and Fumonisin contamination in four maize recombinant inbred families. Toxins (Basel). 2019;11:86. https://doi.org/10.3390/ toxins11020086.

9. Samayoa LF, Cao A, Santiago R, Malvar RA, Butrón A. Genome-wide association analysis for fumonisin content in maize kernels. BMC Plant Biol. 2019;19:1-11

10. Butrón A, Santiago R, Cao A, Samayoa LF, Malvar RA. QTLs for resistance to fusarium ear rot in a multiparent advanced generation intercross (MAGIC) maize population. Plant Dis. 2019:103:897-904

11. Presello DA, Pereyra AO, Iglesias J, Fauguel CM, Sampietro DA, Eyhérabide $\mathrm{GH}$. Responses to selection of S5inbreds for broad-based resistance to ear rots and grain mycotoxin contamination caused by fusarium spp. in maize. Euphytica. 2011;178:23-9.

12. Löffler M, Kessel B, Ouzunova M, Miedaner T. Covariation between line and testcross performance for reduced mycotoxin concentrations in European maize after silk channel inoculation of two fusarium species. Theor Appl Genet. 2011;122:925-34

13. Eller MS, Payne GA, Holland JB. Selection for reduced fusarium ear rot and fumonisin content in advanced backcross maize lines and their topcross hybrids. Crop Sci. 2010;50:2249-60.
14. Bolduan C, Miedaner T, Schipprack W, Dhillon BS, Melchinger AE. Genetic variation for resistance to ear rots and mycotoxins contamination in early European maize inbred lines. Crop Sci. 2009:49:2019-28.

15. Yu J, Holland JB, McMullen MD, Buckler ES. Genetic design and statistical power of nested association mapping in maize. Genetics. 2008;178:539-51.

16. Mackay I, Powell W. Methods for linkage disequilibrium mapping in crops. Trends Plant Sci. 2007;12:57-63.

17. Bernardo R. Bandwagons I, too, have known. Theor Appl Genet. 2016;129:2323-32.

18. Cockram J, Mackay I. Genetic mapping populations for conducting high-resolution trait mapping in plants. Adv Biochem Eng Biotechnol. 2018;164:109-38.

19. Butrón A, Malvar RA, Cartea ME, Ordás A, Velasco P. Resistance of maize inbreds to pink stem borer. Crop Sci. 1999;39:102-7.

20. Jiménez-Galindo JC, Malvar RA, Butrón A, Santiago R, Samayoa LF, Caicedo $M$, et al. Mapping of resistance to corn borers in a MAGIC population of maize. BMC Plant Biol. 2019:19:1-17.

21. Santiago R, Cao A, Malvar RA, Reid LM, Butrón A. Assessment of corn resistance to fumonisin accumulation in a broad collection of inbred lines. F Crop Res. 2013;149:193-202.

22. López-Malvar A, Malvar RA, Butrón A, Revilla P, Pereira-Crespo S, Santiago R. Genetic dissection for maize forage digestibility traits in a multiparent advanced generation intercross (MAGIC) population. Agronomy. 2021;11:104. https://doi.org/10.3390/agronomy11010104.

23. Yi Q, Malvar RA, Álvarez-Iglesias L, Ordás B, Revilla P. Dissecting the genetics of cold tolerance in a multiparental maize population. Theor Appl Genet. 2020;133:503-16.

24. López-Malvar A, Butron A, Malvar RA, McQueen-Mason SJ, Faas L, Gómez $L D$, et al. Association mapping for maize Stover yield and saccharification efficiency using a multiparent advanced generation intercross (MAGIC) population. Sci Rep. 2021;11:1-9. https://doi.org/10.1038/ s41598-021-83107-1.

25. López-Malvar A, Malvar RA, Gomez LD, Barros-rios J, Pereira-crespo S. Elucidating the multifunctional role of the Cell Wall components in the maize exploitation. BMC Plant Biol. 2021:21:251.

26. Huang BE, Verbyla KL, Verbyla AP, Raghavan C, Singh VK, Gaur P, et al. MAGIC populations in crops: current status and future prospects. Theor Appl Genet. 2015;128:999-1017.

27. Zhang X, Pérez-Rodríguez P, Burqueño J, Olsen M, Buckler E, Atlin G, et al. Rapid cycling genomic selection in a multiparental tropical maize population. G3 genes. Genomes Genet. 2017:7:2315-26.

28. Hung HY, Holland JB. Diallel analysis of resistance to fusarium ear rot and fumonisin contamination in maize. Crop Sci. 2012;52:2173-81.

29. Eller MS, Holland JB, Payne GA. Breeding for improved resistance to fumonisin contamination in maize. Toxin Rev. 2008;27:371-89.

30. Clements MJ, Kleinschmidt CE, Pataky JK, White DG. Evaluation of inoculation techniques for fusarium ear rot and Fumonisin contamination of corn. Plant Dis. 2003:87:147-53.

31. Löffler M, Miedaner T, Kessel B, Ouzunova M. Mycotoxin accumulation and corresponding ear rot rating in three maturity groups of European maize inoculated by two fusarium species. Euphytica. 2010;174:153-64.

32. Li X, Zhang Y, Clarke JD, Li Y, Dong X. Identification and cloning of a negative regulator of systemic acquired resistance, SNI1, through a screen for suppressors of npr1-1. Cell. 1999:98:329-39.

33. Yan S, Wang W, Marqués J, Mohan R, Saleh A, Durrant WE, et al. Salicylic acid activates DNA damage responses to potentiate plant immunity. Mol Cell. 2013:52:602-10.

34. Wang L, Chen H, Wang C, Hu Z, Yan S. Negative regulator of E2F transcription factors links cell cycle checkpoint and DNA damage repair. Proc Natl Acad Sci U S A. 2018;115:E3837-45.

35. Rodriguez E, El Ghoul H, Mundy J, Petersen M. Making sense of plant autoimmunity and "negative regulators". FEBS J. 2016;283:1385-91.

36. Rodriguez E, Chevalier J, El Ghoul H, Voldum-Clausen K, Mundy J, Petersen M. DNA damage as a consequence of NLR activation. PLoS Genet. 2018;14:1-17.

37. Sánchez-Rangel D, Sánchez-Nieto S, Plasencia J. Fumonisin B1, a toxin produced by fusarium verticillioides, modulates maize $\beta$-1,3-glucanase activities involved in defense response. Planta. 2012;235:965-78.

38. Santiago R, Cao A, Butrón A, Battilani P. Genetic factors involved in Fumonisin accumulation in maize kernels and their implications in maize 
agronomic management and breeding. Toxins (Basel). 2015;7:3267-96. https://doi.org/10.3390/toxins7083267.

39. Mideros SX, Warburton ML, Jamann TM, Windham GL, Paul Williams W, Nelson RJ. Quantitative trait loci influencing mycotoxin contamination of maize: analysis by linkage mapping, characterization of near-isogenic lines, and meta-analysis. Crop Sci. 2014;54:127-42.

40. Burger HM, Shephard GS, Louw W, Rheeder JP, Gelderblom WCA. The mycotoxin distribution in maize milling fractions under experimental conditions. Int J Food Microbiol. 2013;165:57-64.

41. Oldenburg E, Ellner F. Distribution of disease symptoms and mycotoxins in maize ears infected by fusarium culmorum and fusarium graminearum. Mycotoxin Res. 2015;31:117-29.

42. Cao A, Butrón A, Ramos AJ, Marín S, Souto C, Santiago R. Assessing white maize resistance to fumonisin contamination. Eur J Plant Pathol. 2014;138:283-92.

43. Holland JB, Nyquist WE, Cervantes-Martínez CT. Estimating and interpreting heritability for plant breeding: an update. Plant Breed Rev. 2003;22:9-112.

44. Holland JB. Estimating genotypic correlations and their standard errors using multivariate restricted maximum likelihood estimation with SAS Proc MIXED. Crop Sci. 2006;46:642-54.

45. Liu X, Huang M, Fan B, Buckler ES, Zhang Z. Iterative usage of fixed and random effect models for powerful and efficient genome-wide association studies. PLoS Genet. 2016;12:e1005767. https://doi.org/10.1371/ journal.pgen.1005767.

46. Bradbury PJ, Zhang Z, Kroon DE, Casstevens TM, Ramdoss Y, Buckler ES. TASSEL: software for association mapping of complex traits in diverse samples. Bioinforma Appl. 2007;23:2633-5. https://doi.org/10.1093/bioin formatics/btm308.

47. Harper LC, Schaeffer ML, Thistle J, Gardiner JM, Andorf CM, Campbell DA, et al. The MaizeGDB genome browser tutorial: one example of database outreach to biologists via video. Database. 2011;2011:1-7.

\section{Publisher's Note}

Springer Nature remains neutral with regard to jurisdictional claims in published maps and institutional affiliations.

Ready to submit your research? Choose BMC and benefit from:

- fast, convenient online submission

- thorough peer review by experienced researchers in your field

- rapid publication on acceptance

- support for research data, including large and complex data types

- gold Open Access which fosters wider collaboration and increased citations

- maximum visibility for your research: over $100 \mathrm{M}$ website views per year

At BMC, research is always in progress.

Learn more biomedcentral.com/submissions 